\title{
Hückel: \\ Um Programa Didáctico para Computadores Pessoais de Sistema Operativo MS/DOS
}

\author{
PAULO SANCHES E ANDRÉ MELO**
}

\section{O método de Hückel possibilita uma razoável compreensão da estrutura electrónica e de muitas propriedades de sistemas conjugados planares. O programa "Hückel" permite efectuar cálculos deste tipo usando computadores pessoais de sistema operativo MS/DOS. Este programa funciona de uma forma interactiva oferecendo ao utilizador um leque bastante alargado de opções de execução.}

\section{INTRODUÇÃ̃O}

A resolução exacta da equação de Schrödinger só é possivel para sistemas monoelectrónicos ou para sistemas modelo como a partícula na caixa, 0 rotor rígido ou o oscilador harmónico. Em sistemas atómicos ou moleculares polielectrónicos é necessário recorrer a métodos aproximados baseados normalmente na teoria das perturbações ou no formalismo variacional. Neste contexto, existem diversos métodos que permitem a resolução aproximada da equação de Schrödinger e que contribuem para uma melhor compreensão da estrutura e reactividade química. 0 método de Hückel, aplicado no estudo da estrutura $\pi$ de sistemas conjugados planares, é o método mais simples deste tipo.

Na secção 2 faz-se uma breve abordagem do método de Hückel bem como dos indices moleculares mais frequentemente calculados de acordo com o seu formalismo.

Com base neste método Cordeiro e Gomes $^{1}$ desenvolveram um programa para computadores pessoais da série Apple, entretanto caídos em desuso. Na continuação deste trabalho desenvolvido no nosso grupo, foi elaborado um novo programa, designado por "Hückel", para computadores pessoais de sistema operativo MS/DOS. A sua descrição é apresentada na secção 3. Nesta secção, são também referidas as opções adicionais de cálculo introduzidas em relação ao programa anterior.

\section{MÉTODO DE HÜCKEL}

0 método de Hückel foi proposto por Erich Hückel ${ }^{2}$ em 1931 para o estudo da estrutura electrónica $\pi$ de hidrocarbonetos conjugados planares.

Este método admite que os electrões $\pi$ e $\sigma$ podem ser estudados separadamente, apenas tratando posteriormente os primeiros. A razoabilidade desta aproximação resulta de, nos sistemas moleculares considerados, ser proibida por simetria a combinação entre orbitais $\pi$, antissimétricas em relação à reflexão no plano da molécula, e as orbitais $\sigma$, simétricas em relação a esta operação de simetria.

De acordo com a aproximação considerada, num sistema conjugado com $\kappa$ electrões (m electrões $\pi$ e $\kappa$-m electrões $\sigma$ ) 0 hamiltoniano electrónico total $\hat{H}_{e l}(1, \ldots, m, \ldots, k)$ pode ser escrito como a soma de duas parcelas independentes:

$\hat{H}_{e \mid}(1, \ldots, m, \ldots, k)=\hat{H}_{\pi}(1, \ldots, m)+\hat{H}_{\sigma}(m+1, \ldots, k)$

Consequentemente a função de onda total $\Psi(1, \ldots, \mathrm{m}, \ldots, \mathrm{k})$ pode ser escrita como o produto de duas funções independentes:

$\Psi(1, \ldots, \mathrm{m}, \ldots, \mathrm{k})=\Psi_{\pi}(1, \ldots, \mathrm{m}) \Psi_{\sigma}(\mathrm{m}+1, \ldots, \mathrm{k})$

e a energia electrónica total $E_{e \mid}$ como a soma de dois termos distintos:

$\mathrm{E}_{\mathrm{el}}=\mathrm{E}_{\pi}+\mathrm{E}_{\mathrm{o}}$

Na resolução da equação de Schrödinger relativa aos $m$ electrões $\pi$ do sistema,

$$
\hat{H}_{\pi}(1, \ldots, m) \quad \Psi_{\pi}(1, \ldots, m)=E_{\pi} \Psi_{\pi}(1, \ldots, m)
$$

são feitas as seguintes aproximações:

- 0s electrões $\pi$ são considera- dos independentes, o que implica que 0 hamiltoniano $\hat{H}_{\pi}$ pode ser expresso como um somatório de componentes monoelectrónicas, $\hat{h}_{\pi}(i)$,

$$
\hat{H}_{\pi}(1, \ldots, m)=\sum_{i=1}^{m} \hat{h}_{\pi}(i)
$$

e a função de estado electrónica $\Psi_{\pi}$ possa ser escrita como 0 produto de orbitais moleculares (OM) monoelectrónicas, $\phi_{i}(i)$,

$$
\Psi_{\pi}(1, \ldots, m)=\prod_{i=1}^{m} \phi_{i}(i)
$$

- As OM são representadas como combinações lineares de um conjunto de $n$ orbitais atómicas $(O A) \chi p$,

$$
\phi_{i}=\sum_{p=1}^{m} C_{p i} \chi p
$$

este conjunto, contém uma $O A p_{z}$ por cada átomo que participa no sistema $\pi \mathrm{e}$ é considerado ortonormal $\left(\mathrm{S}_{\mathrm{pr}}=\int \chi_{\mathrm{p}}{ }^{*} \chi_{\mathrm{r}}\right.$ $\left.\mathrm{d} \tau=\delta_{\mathrm{pr}}\right)$.

Atendendo à primeira simplificação, a equação (4), equação de valores próprios do operador $\hat{H}_{\pi}(1, \ldots, m)$, é equivalente ao seguinte sistema de equações monoelectrónicas (equações de Hückel):

$$
\hat{h}_{\pi}(i) \phi_{i}(i)=\varepsilon_{i} \phi_{i}(i)
$$

Atendendo à segunda simplificação, a aplicação do método variacional à resolução aproximada deste problema conduz a um conjunto de equações do tipo:

$$
\sum_{p=1}^{n} c_{p i}\left(h_{p r}^{\pi}-\varepsilon_{i} S_{p r}\right)=0 \quad \begin{aligned}
& r=1,2 \ldots, n \\
& i=1,2, \ldots, n
\end{aligned}
$$

ou

$$
\stackrel{\mathrm{h}}{\approx} \underset{\approx}{\mathrm{C}}=\underset{\approx}{\mathrm{C}}
$$




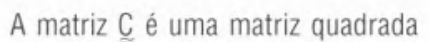
$(n X n)$ cujos elementos $C_{p i}$ são os coeficientes das $\mathrm{OA} \chi_{p}$ para as $\mathrm{OM} \phi_{i}$.

A matriz $\mathrm{h}_{\approx} \pi$ é uma matriz quadrada ( $\mathrm{X} \mathrm{n})$, designada por matriz de Hückel, cujos elementos $h_{p r}^{\pi}$ são definidos como:

$$
h_{p r}^{\pi}=\int \chi_{p}{ }^{*} \hat{h}_{\pi} \chi_{s} d \tau
$$

Os valores próprios desta matriz são as energias $\varepsilon_{\mathrm{i}}$ das $0 \mathrm{M}$ que podem ser calculadas pela expressão,

$$
\varepsilon_{i}=\sum_{p=1}^{n} c_{p i}^{\star} c_{p i} h_{p p}^{\pi}+\sum_{p=1}^{n} \sum_{r=1}^{n} c_{p i}^{\star} c_{r i} h_{p r}^{\pi}
$$

e constituem os elementos não nulos da matriz diagonal $(n \times n) \varepsilon$.

A energia total $\pi$ do sistema é dada por,

$$
\begin{gathered}
E_{\pi}=\sum_{i=1}^{n} n_{i} \varepsilon_{i}=\sum_{i=1}^{n} n_{i} \\
\left(\sum_{p=1}^{n} c_{p i}^{*} c_{p i} h_{p p}^{\pi}+\sum_{p=1}^{n} \sum_{i=1}^{n} c_{p i}^{*} c_{r i} h_{p r}^{\pi}\right)
\end{gathered}
$$

onde $n_{\mathrm{i}}$ é a ocupação electrónica da $\mathrm{OM} \phi_{\mathrm{i}}$.

Os integrais $h_{p r}^{\pi}$ não são calculados analiticamente, sendo igualados a parâmetros empíricos:

$h_{p p}^{\pi}=\alpha$ se p for um átomo de carbono.

$h_{p r}^{\pi}=\beta$ se $p \neq r$, sendo $p$ e $r$ dois átomos

de carbono ligados

$h_{p r}^{\pi}=0$ se $p \neq r$, sendo $p$ e r átomos não ligados.

Quando existem heteroátomos no sistema $\pi$, por exemplo flúor, cloro, bromo, boro, azoto ou oxigénio, são adoptados valores diferentes para estes integrais. Assim se 0 átomo $p$ for um heteroátomo X e r um átomo de carbono ligado a $p$,

$h_{p p}^{\pi}=\alpha+h_{\chi} \beta$
$h_{p r}^{\pi}=K_{C-\chi} \beta$

onde $\mathrm{h}_{\chi}$ e $\mathrm{K}_{\mathrm{C}-\chi}$ são parâmetros característicos do heteroátomo ${ }^{3}$.

Para 0 átomo de enxofre o tratamento efectuado, de modo a reflectir de algum modo a contribuição das orbitais 3d, é um pouco diferente do anterior ${ }^{3}$. Assim, cada átomo de enxofre (S) é desdobrado em dois átomos (S1 e S2), admitindo-se que cada um deles contribui com um electrão para 0 sistema $\pi$.

Foram também propostas alterações do modelo inicial para o tratamento de grupos metilo. Três modelos foram considerados para este fim ${ }^{3}$.

- Modelo indutivo - Assume-se que 0 grupo metilo não participa no sistema $\pi$ mas exerce um efeito indutivo sobre 0 carbono $\left(C_{\alpha}\right)$ ao qual está ligado. De acordo com esta aproximação 0 referido átomo de carbono $\left(\mathrm{C}_{\alpha}\right)$ é tratado como um heteroátomo.

- Modelo do heteroátomo - Assume-se que 0 grupo metilo participa no sistema $\pi$ como uma entidade única contribuindo para este com dois electrões. De acordo com esta aproximação o grupo metilo é encarado como um heteroátomo.

- Modelo conjugativo - Assume-se que 0 grupo metilo participa no sistema $\pi$ como duas entidades, 0 carbono (C) e os hidrogénios $\left(\mathrm{H}_{3}\right)$, reflectindo assim 0 efeito conjugativo. De acordo com esta aproximação as duas entidades referidas são tratadas como heteroátomos contribuindo cada uma com um electrão para 0 sistema $\pi$.

Atendendo às aproximações (14), o sistema de equações (10) pode ser reescrito da seguinte forma,

ou

$$
\begin{array}{ll}
\sum_{i=1}^{n} C_{p i}\left(h_{p r}^{t o p}-y_{i}\right)=0 & \begin{array}{l}
r=1,2 \ldots, n \\
i=1,2, \ldots, n
\end{array}
\end{array}
$$

$$
\mathrm{h}^{\text {top }} \mathrm{C}=\mathrm{C} \underset{\mathrm{Y}}{\cong}
$$

onde $Y_{i}=\left(\varepsilon_{i}-\alpha\right) / \beta$ e h top é a matriz topológica de Hückel cujos elementos são dados por:

$\mathrm{h}_{\mathrm{pp}}^{\mathrm{top}}=0$ se $\mathrm{p}$ for um átomo de carbono.

$h_{p r}^{\text {top }}=-1$ se $p \neq r$, sendo $p$ e r átomos de carbono ligados entre si.

$h_{p p}^{\text {top }}=-h_{\chi}$ se $p$ for um heteroátomo $\chi$.

$\mathrm{h}_{\mathrm{pr}}^{\mathrm{top}}=-\mathrm{K}_{\mathrm{C}-\chi}$ se $\mathrm{p} \neq \mathrm{r}$, sendo $\mathrm{p}$ um átomo de carbono e r um heteroátomo $\mathrm{X}$. ligados entre si.

$\mathrm{h}_{\mathrm{pr}}^{\text {top }}=0$ se $\mathrm{p} \neq \mathrm{r}$, sendo $\mathrm{p}$ e $\mathrm{r}$ átomos não ligados.

Diversos índices moleculares são calculados dentro do formalismo de
Hückel. Apresentam-se abaixo os mais importantes destes índices.

— Carga electrónica $\pi$ do átomo $r$

$$
\mathrm{q}_{\mathrm{r}}=\sum_{\mathrm{i}=1}^{\mathrm{n}} \mathrm{n}_{\mathrm{i}} \mathrm{c}_{\mathrm{ri}}^{*} \mathrm{c}_{\mathrm{ri}}
$$

— Ordem de ligação $\pi$ entre os átomos res

$$
P_{r s}=\sum_{i=1}^{n} n_{i} c_{r i}^{\star} c_{s i}
$$

A ordem de ligação entre dois átomos dá uma medida da força da respectiva ligação $\pi$.

Com base neste indice, Coulson ${ }^{4}$ propôs uma fórmula empírica para calcular o comprimento da ligação (C-C) entre os átomos $\mathrm{r}$ e $\mathrm{s}$.

$$
R_{r s}=S_{C-c}-\frac{S_{c-c}-d_{c-c}}{1+K\left(1-P_{r S}\right) / P_{r s}}
$$

em que $S_{c-c}$ é o comprimento de uma

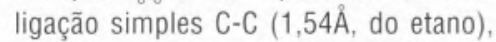
$\mathrm{d}_{\mathrm{c}-\mathrm{c}}$ é 0 comprimento de uma ligação dupla $\mathrm{C}=\mathrm{C}(1,34 \hat{A}$, do etileno) e $\mathrm{K}$ é um parâmetro empírico.

— Valência livre de um átomo r

$$
F_{r}=\sqrt{3}-\sum_{s}^{\text {ligados a r }} \text { Prs }
$$

Este índice, dá uma medida da carga residual $\pi$ dos átomos de carbono disponivel para formar novas ligações e é calculado como a diferença entre a capacidade máxima de ligação dum átomo de carbono( $\sqrt{3}$ no átomo central do trimetilmetano) e a capacidade ligante do átomo considerado.

- Polarizabilidade atómica

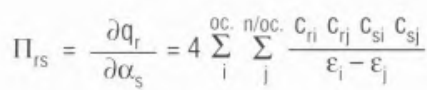

Este índice reflecte a mudança que ocorre na densidade de carga $\pi$ de um átomo $\mathrm{r}$ em consequência de uma alteração unitária da densidade de carga $\pi$ do átomo $\mathrm{s}$. 


\section{ESTRUTURA DO PROGRAMA "HÜCKEL"}

0 programa "Hückel" foi escrito na linguagem de programação Fortran 77 e pode ser executado em computadores pessoais de sistema operativo MS/DOS

Este programa é constituido por um programa principal e catorze subrotinas que se interligam como se mostra na figura 1.

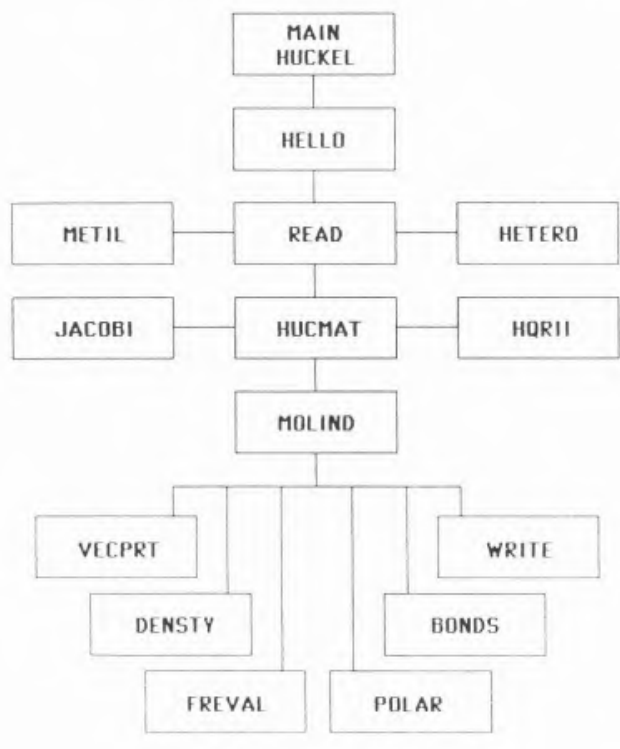

Figura 1. Fluxograma do programa "Hückel".

As unidades deste programa têm as seguintes funções:

MAIN HUCKEL - Controla a entrada e saída do programa.

Subrotina HELLO - Corre automaticamente quando o programa é executado fazendo a apresentação do mesmo. Mostra, em seguida, um menu que permite controlar as principais opções de execução do programa.

Subrotina READ - Lê as informações topológicas da molécula necessárias à construção da matriz topológica de Hückel.

Subrotina METIL - Introduz e caracteriza os grupos metilo.

Subrotina HETERO - Caracteriza os heteroátomos.

Subrotina HUCMAT - Constrói e permite visualizar a matriz topológica de Hückel.

Subrotina JACOBI - Determina os valores e vectores próprios da matriz topológica de Hückel, utilizando o método de diagonalização de Jacobi ${ }^{5}$.

Subrotina HaRII (elaborada por Beppu e Ninomiya ${ }^{6}$ ) - Determina os valores e vectores próprios da matriz topológica de Hüc-

Tabela 1 - Tempo gasto na determinação dos vectores e valores próprios da matriz topológica de Hückel para várias espécies químicas.

\begin{tabular}{lclll}
\hline Espécie química & $\mathbf{n}^{\mathbf{0}}$ de átomos de carbono & $\mathbf{t}_{\mathbf{j}} \mathbf{1}(\mathbf{s})$ & $\mathbf{t}_{\mathbf{j}} \mathbf{2}(\mathbf{s})$ & $\mathbf{t}_{\mathrm{H}_{2}}(\mathbf{s})$ \\
\hline Pentadienilo & 5 & 76 & 2,4 & 1,5 \\
Benzeno & 6 & 149 & 2,9 & 2,0 \\
Heptatrienilo & 7 & 187 & 5,3 & 2,8 \\
Octatetraeno & 8 & 293 & 7,8 & 3,2 \\
Benzociclopentadienilo & 9 & 386 & 11,3 & 4,6 \\
Bifenilo & 12 & 723 & 28,1 & 6,5 \\
1-Femalenilo & 13 & 2530 & 32,2 & 7,8 \\
\hline
\end{tabular}

$t_{1}$ tempo gasto utilizando o método de Jacobi e o programa elaborado por Cordeiro e Gomes.

$t_{12}$ tempo gasto utilizando o método de Jacobi e o presente programa.

$t_{\mathrm{H} 2}$ tempo gasto utilizando o método de Householder e o presente programa.

kel, utilizando 0 método de tridiagonalização de Householder ${ }^{7}$.

Subrotina MOLIND - Controla o cálculo das propriedades moleculares, apresentando um menu que permite escolher a propriedade pretendida.

Subrotina VECPRT - Imprime os valores próprios (energias das $0 \mathrm{M}$ ) e vectores próprios (coeficientes das 0 M) da matriz de Hückel e a energia total $\pi$.

Subrotina DENSTY - Calcula e imprime as cargas electrónicas $\pi$ de todos os átomos da molécula.

Subrotina FREVAL - Calcula e imprime as valências livres dos átomos pertencentes à molécula desde que 0 sistema não tenha heteroátomos nem grupos metilo.

Subrotina POLAR - Calcula e imprime as polarizabilidades $\pi$ para todos os pares de átomos em sistemas de camada fechada.

Subrotina BONDS - Imprime as ordens de ligação p para todas as ligações da molécula. Em hidrocarbonetos sem grupos metilo nem heteroátomos são também calculados os comprimentos de ligação de Coulson.

Subrotina WRITE - Escreve todas as propriedades moleculares num ficheiro com o nome da molécula e extensāo .RES.

0 presente programa "Hückel" apresenta as seguintes opcões adicionais de cálculo relativamente ao elaborado por Cordeiro e Gomes ${ }^{1}$.

i) Tratamento dos heteroátomos ( $\mathrm{B}, \mathrm{F}, \mathrm{Cl}$ e Br).

ii) Tratamento dos grupos metilo utilizando três modelos alternativos (indutivo, de heteroátomo e conjugativo).

iii) Possibilidade da determinação dos vectores e valores próprios da matriz topológica de Hückel, utilizando 0 algoritmo de tridiagonalização de Householder. Este algoritmo apresenta vantagens significativas sobre 0 algoritmo de diagonalizacão de Jacobi principalmente para espécies químicas com muitos átomos. iv) Possibilidade do cálculo dos comprimentos de ligacão de Coulson em hidrocarbonetos sem grupos metilo nem heteroátomos.

0 programa "Hückel" aqui apresentado foi testado para uma série de moléculas, tendo os cálculos sido efectuados num computador pessoal Unisys / 286. Foram utilizados os dois métodos atrás indicados (Jacobi e Householder) para a determinação dos vectores e valores próprios da matriz topológica de Hückel.

Na tabela 1, são comparados os tempos gastos nesta determinação com os obtidos utilizando o programa elaborado por Cordeiro e Gomes ${ }^{1}$ num computador pessoal Apple IIe.

- Departamento de Quimica

\section{REFERÊNCIAS}

1. M. Natália D.S. Cordeiro e J.A.N.F. Gomes, Bol. Soc. Port. Quim., 26 (1986) 33.

2. E. Hückel, Z. Physik, 72 (1931) 310.

3. A. Streitweiser, Molecular Orbital Theory, John Wiley \& Sons, New York, 1962.

4. C.A. Coulson, Proc. Roy. Soc., London, A169 (1939) 413

5. A. R. Gourbuy e G. A. Watson, Computational Methods for Matrix Eigenproblems, John Wiley \& Sons. New York, 1973.

6. Y. Beppu e I. Ninomiya, Computers $\S$ Chemistry, 6 (1982) 87

7. K. J. Bathe e E. Wilson, Numerical Methods in Finite Element Analysis, Prentice-Hall, New Jersey, 1976 


\section{GERB $R$ \\ INSTRUM NTS}

\section{RKIN ELMER}

(RM)TAMSON

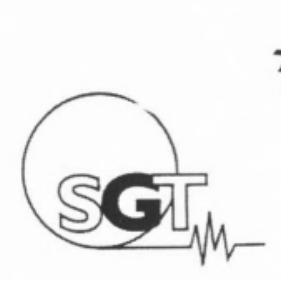

\#馬

METTLER TOLEDO

FisherScientific

KOJAIR

\section{METTLR TOLEDO}

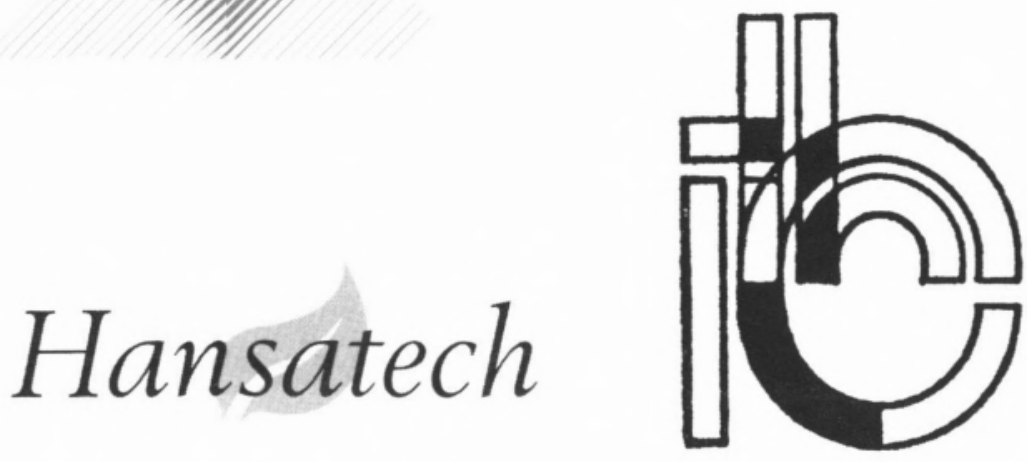

\section{$\triangle$ GOCOAEX}

A \& R DESIGNS LTD

\section{IE C CRISTN I}

\section{Alliech}

UNIEQUIP
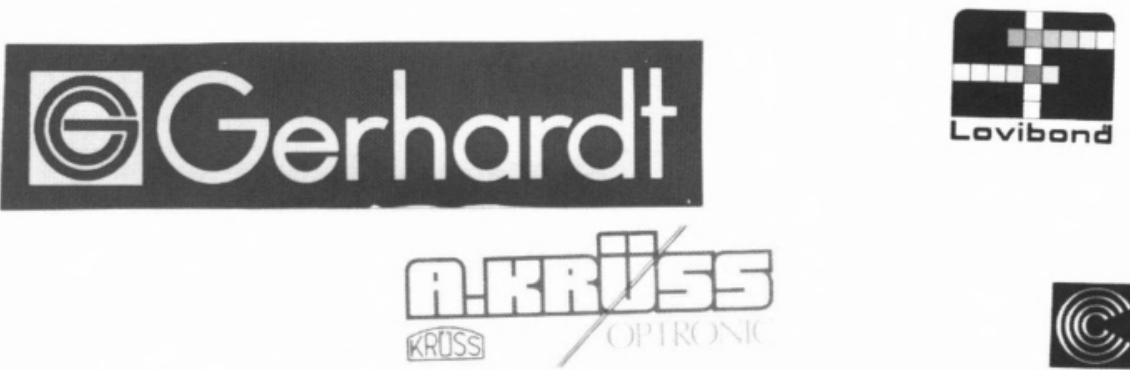

Promochem

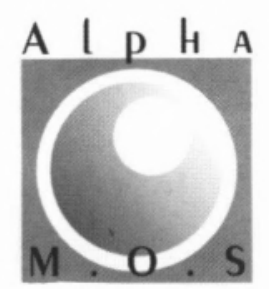

\section{CONSORT}

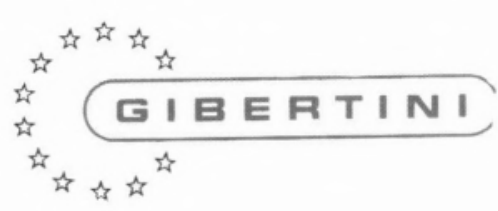

inftrumentos de laboratório e científicos lda

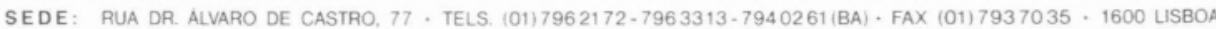
AV. DA BOAVISTA, 80, 3., SALA 29 . TELEF. 6097503 . FAX 6003154 . 4000 PORTO

FILIAIS: RUA MACHADO DOS SANTOS, $61-63$ TELEF. 27043.9500 PONTA DELGADA-AÇORES RUA PADRE ESTEVÁO CABRAL, 79, 3., SALA 311 \% TEL./FAX (039)35022:3000 COIMBRA 


\section{ESPECTROFOTOMETROS SPECTRONIC ${ }^{\circledR}$ GENESYS $^{\text {TM }}$ CARACTERÍSTICAS NOTÁVEIS, PREÇOS RAZOÁVEIS... E AGORA, MAIS}

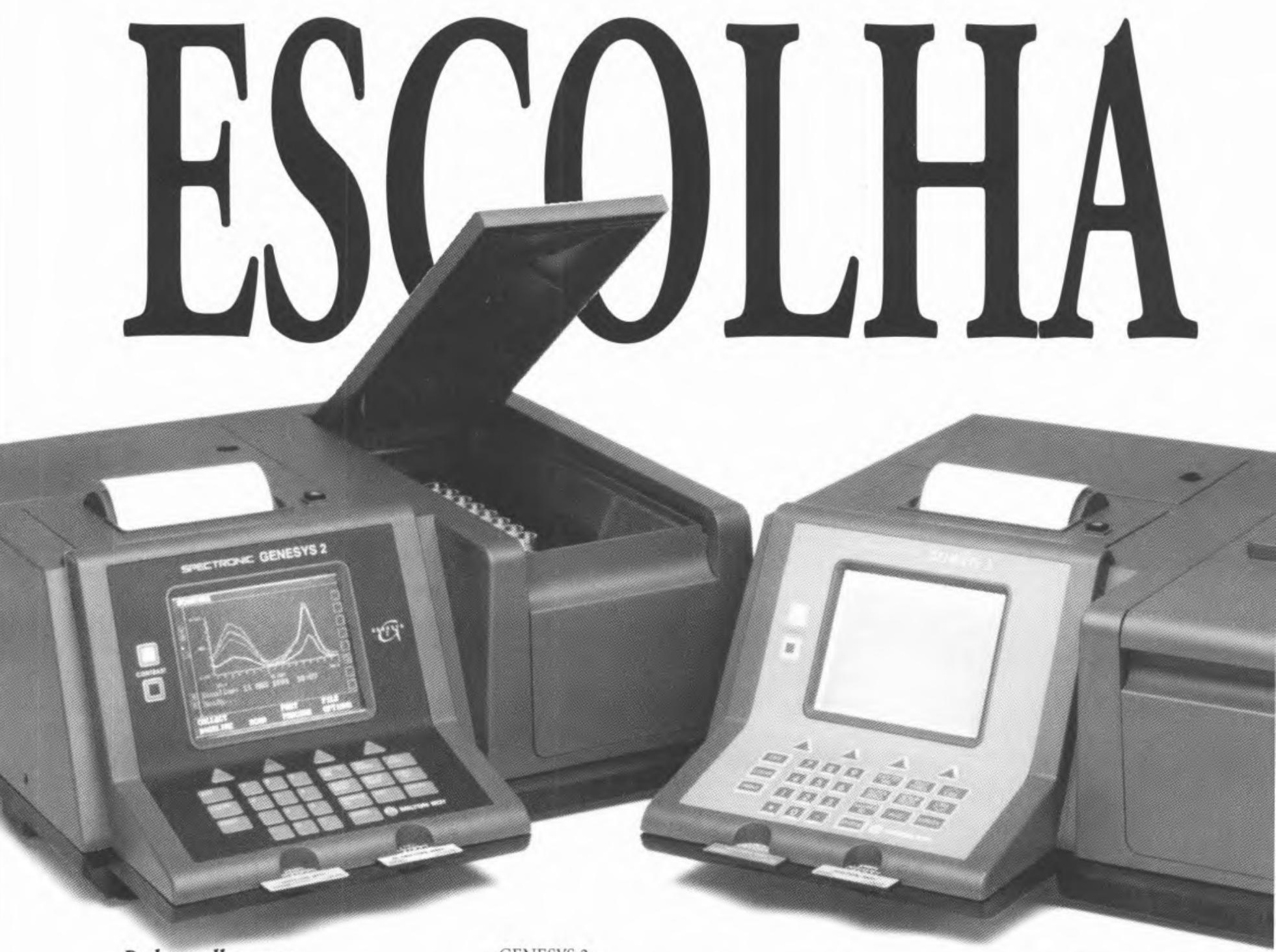

\section{Pode escolher entre}

GENESYS 5

Espectrofotómetros de desenho moderno, com um sistema óptico único, de elevada precisão e fiabilidade, baseado num feixe duplo e dois detectores (um para a amostra e outro para 0 padrão); de fácil utilização, écran gráfico com ajuste de contraste e possibilidade de utilização de disquetes para memorizaçáo de testes, curvas de calibração e diversos programas analíticos.

- Comprimento de onda: 200 - $1100 \mathrm{~nm}$, com precisăo de $+/-1 \mathrm{~nm}$ e exactidāo de $+l-0,5 \mathrm{~nm}$; largura de banda de $5 \mathrm{~nm}$. - Velocidade de varrimento: $300 \mathrm{~nm} / \mathrm{min}$. ou $900 \mathrm{~nm} / \mathrm{min}$.

- Suporte para 8 cuvetes e interface RS 232C para computador e saída centronics para impressora externa.
GENESYS 2

Semelhante ao anterior, mas com largura de banda de $2 \mathrm{~nm}$, écran gráfico colorido, velocidade de varrimento programável até $2400 \mathrm{~nm} / \mathrm{min}$. e disquete para memorizaçāo de testes incluída.

ACESSÓRIOS COMUNS AOS 2 MODELOS

- Disquete para memorizaçáo de testes com 128K;

- Disquete para memorizaçáo de testes com $256 \mathrm{~K}$

- Disquete "Application I" com programas para varrimento, cinética, razẫo de absorvância, curva padrāo, etc;

- Disquete "Application II", inclui todos os programas da disquete anterior, mais: cinética avançada, múltiplo comprimento de onda $\mathrm{e}$ diferencial de absorvâncias;

- Disquete "Performance Validation", para uso com os padrōes "Spectronic", que permite testar a performance do aparelho e calibrá-lo.
Peça-nos Catálogos e Preços

EMÍLIO DE AZEVEDO

CAMPOS \& CA., LDA.

R. Senhora da Penha, 110-114

4461 Sra. da Hora

Telef.: (02) 9531183

Fax: (02) 9531430

R. Antero Quental, 17 - $1^{\circ}$

1150 Lisboa

Telef.: (01) 3520194

Fax: (01) 353382 